\title{
Therapeutic Management of Pyometra with Methylergometrine and Cabergoline in Bitches - An Alternative Approach
}

\author{
A. Ganesan, D. Antoine ${ }^{1}$, A. Palanisammi, R. Ramprabu
}

10.18805/IJAR.B-4721

\begin{abstract}
Background: Canine pyometra is a common reproductive disorder of sexually intact bitches characterized by accumulation of pus in the uterine lumen and it is always associated with vagaries of clinical and pathological manifestations of multisystemic origin.

Methods: The present study involved twenty three bitches of 8-13 years of age with pyometra. Diagnosis of pyometra was performed based on the anamnesis, ultrasound examination, hematoglocial and biochemical changes. As an alternate to conventional prostaglandin protocol, Tab. Methylergometrine $0.125 \mathrm{mg} / \mathrm{dog}$ (Methergine, Novartis) BID and Tab. Cabergoline @ $10 \mu \mathrm{g} / \mathrm{kg}$ body weight (Cabgolin, Sun Pharma) SID and Tab. Amoxicillin- Clavulinic acid @ $10 \mathrm{mg} / \mathrm{kg}$ body weight (Augmentin, Glaxo Smithkline) BID was advised for two weeks. In addition to that supportive fluid therapy and Inj. Pantoprazole @ $1 \mathrm{mg} / \mathrm{kg}$ body weight was also administered prior to initiation of treatment protocol. There was a significant clinical recovery in terms of physiological, hematological and biochemical parameters. Ultrasound examination on Day $0,7,9$ and Day 14 of treatment revealed reduction in the diameter of uterine sacculations. All the bitches recovered after 10 to 15 days with complete evacuation of pus, with no recurrence.

Result: Our investigation revealed that this alternative protocol along with antibiotics and supportive fluid therapy can be a better alternative to young dogs to preserve the reproductive capability and also in geriatric dogs which are unfit for traditional ovariohysterectomy.
\end{abstract}

Key words: Canine pyometra, Diestrus, Prostagalndins.

\section{INTRODUCTION}

Canine pyometra is an acute or chronic inflammation cum suppurative infection of uterus exemplified by accumulation of pus in the lumen (Smith, 2006). This reproductive disorder is mainly due to an upshot of physiological effects of progesterone and often ovariohysterectomy is considered to be the best empirical therapy as a part of both preventive and curative measures for recurrence of pyometra (Kumar et al., 2010). Indications for medical management of pyometra includes patent cervix, significant reproductive value intended for breeding and absence of reproductive emergencies (Andersen and Farstad, 2006) (Shukla, 2012). Young bitches with open cervix pyometra, normal organ functions and predictable breeding values are the candidature for Medical/Prostaglandin therapies (Corrada et al., 2006; Sandhya, 2018). On the other hand mid to old aged dogs with systemic inflammatory responses with compromised organ functions often unfit for surgery/medical management owing to its age factor, anesthetic and high risk for serious complications or even death (Burke,1982; Shiju Simon et al., 2011). Hence devising a protocol to mimic prostaglandin therapy is highly imperative for ensuing evacuation of pus and achieving luteolysis.

\section{MATERIALS AND METHODS}

The present study included twenty three bitches of 8-13 years of age with open cervix pyometra. Diagnosis of open cervix pyometra was performed based on the anamnesis, ultrasound examination, hematoglocial and biochemical
Department of Veterinary Gynaecology and Obstetrics, Veterinary Clinical Complex, Veterinary College and Research Institute, Tamil Nadu Veterinary and Animal Sciences University, Tirunelveli-627 358, Tamil Nadu, India.

'Department of Veterinary Gynaecology and Obstetrics, Rajiv Gandhi College of Veterinary and Animal Sciences, Puducherry605 009, Tamil Nadu, India.

Corresponding Author: A. Ganesan, Department of Veterinary Gynaecology and Obstetrics, Veterinary Clinical Complex, Veterinary College and Research Institute, Tamil Nadu Veterinary and Animal Sciences University, Tirunelveli-627 358, Tamil Nadu, India. Email: ganvet43@gmail.com

How to cite this article: Ganesan, A., Antoine, D., Palanisammi, A. and Ramprabu, R. (2021). Therapeutic Management of Pyometra with Methylergometrine and Cabergoline in Bitches - An Alternative Approach. Indian Journal of Animal Research. DOI: 10.18805/ IJAR.B-4721.

Submitted: 14-07-2021 Accepted: 11-10-2021 Online: 03-11-2021

changes. Before treatment and nine days after treatment, blood samples with and without anticoagulant were collected from all the bitches for estimation of haematological and biochemical parameters. As an alternate to conventional prostaglandin protocol, Tab. Methylergometrine $0.125 \mathrm{mg} /$ dog (Methergine, Novartis) BID and Tab. Cabergoline @ 10 $\mu \mathrm{g} / \mathrm{kg}$ body weight (Cabgolin, Sun Pharma) SID and Tab. Amoxicillin- Clavulinic acid @ $10 \mathrm{mg} / \mathrm{kg}$ body weight (Augmentin, Glaxo Smithkline) BID was advised for seven days. In addition to that supportive fluid therapy and Inj. 
Pantoprazole @ 1mg/kg body weight was also administered prior to initiation of treatment protocol. Ultrasonography of the uterus was performed on day $0,7,9$ and day 14 of the treatment. The data obtained from the above study were analyzed Wilcoxon matched-pairs signed rank test (Dabhi and Dhami, 2006).

\section{RESULTS AND DISCUSSION}

After treatment protocol twenty one bitches recovered uneventfully wherein two bitches died on day 2 and 4 after treatment due to severe systemic inflammatory responses syndrome (SIRS). A mild normocytic, normochromic non degenerative anemia was observed at day 0 of treatment however, there was a significant increase in the total erythrocyte count at day 9 due to restoration of partial appetite and reduction in the endotoxemic effects on bone marrow (Baithalu et al., 2010). The common clinical finding in pyometra was elevated levels of mean TLC of about $36.9 \pm 6.910^{3} \mu$ indicating acute inflammatory responses and after 9 days of treatment protocol there was significant restoration of TLC to physiological limits to $16.86 \pm 1.310^{3} \mu \mathrm{l}$ as mentioned in the Table 1 . There was a significant reduction in the blood urea nitrogen (BUN) and creatinine values at day 9 and 15 after treatment which concurred with the findings of Kantharaj et al. (2018).

Ultrasonographical evaluation of uterine sacculations was measured in the present study on day 0 (before the start of treatment), day 7,9 and on day 14 . The characteristic hypo to anechoic sacculations was measured by making longitudinal and cross sections of uterus (Fig 1-4). It was observed from our study that there was significant $(P<0.01)$ reduction in the uterine sacculations from $31.04 \pm 4.5 \mathrm{~mm}$ on day 0 to $16 \pm 4.2 \mathrm{~mm}$ on day 7 and there was complete evacuation of pus at day 9; however in bitches having severe distention and uterine sacculation diameter of more than $20 \mathrm{~mm}$ took more than 13.8 days (average) for complete evacuation of pus (Fig 5 and 6). On the other hand bitches with $5-10 \mathrm{~mm}$ diameter uterine sacculations took 9 days (average) for complete evacuation of pus. From our study it was observed that for complete evacuation of pus in open

Table 1: Results of serum biochemical constituents before and after treatment.

\begin{tabular}{lccc}
\hline $\begin{array}{l}\text { Biochemical } \\
\text { Parameters }\end{array}$ & Day 0 & Day 9 & Day 15 \\
\hline $\mathrm{Hb}$ & $11.87 \pm 1.15$ & $12.56 \pm 1.7$ & $14.7 \pm 0.9$ \\
$\mathrm{PCV}$ & $30.7 \pm 3.5$ & $33.56 \pm 4.0$ & $35.17 \pm 2.0$ \\
$\mathrm{TEC}$ & $5.8 \pm 0.5$ & $6.8 \pm 0.7$ & $7.5 \pm 0.9$ \\
$\mathrm{TLC}$ & $36.9 \pm 6.9$ & $16.86 \pm 1.3$ & $10.9 \pm 1.6$ \\
$\mathrm{Neutrophils}$ & $87.5 \pm 1.6$ & $82.5 \pm 1.8$ & $78.5 \pm 1.5$ \\
$\mathrm{Na}$ & $130.9 \pm 9.6$ & $143.4 \pm 6.0$ & $145.4 \pm 3.0$ \\
$\mathrm{Cl}$ & $108 \pm 2.5$ & $118 \pm 2.4$ & $138 \pm 1.9$ \\
$\mathrm{~K}$ & $5.62 \pm 0.12$ & $3.62 \pm 0.44$ & $4.0 \pm 0.6$ \\
$\mathrm{BUN}$ & $119 \pm 9.7$ & $26.5 \pm 1.0$ & $9.5 \pm 2.0$ \\
Creatinine & $2.0 \pm 0.05$ & $0.8 \pm 0.03$ & $0.5 \pm 0.03$ \\
\hline
\end{tabular}

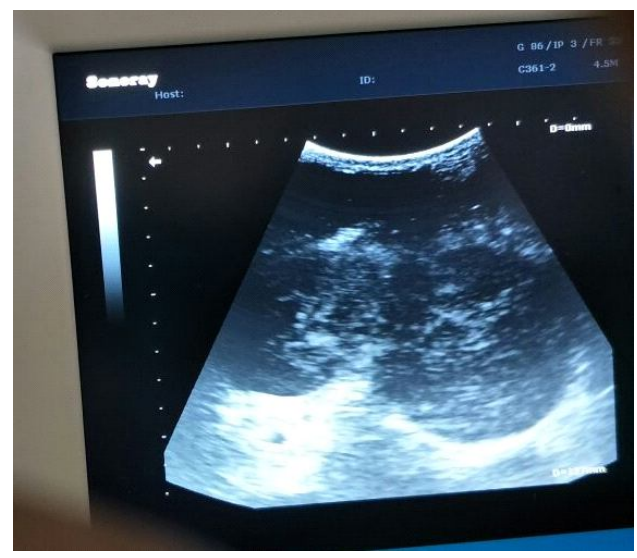

Fig 1: Cross section of distinct hypoechoic uterine sacculations characteristic of pyometra.

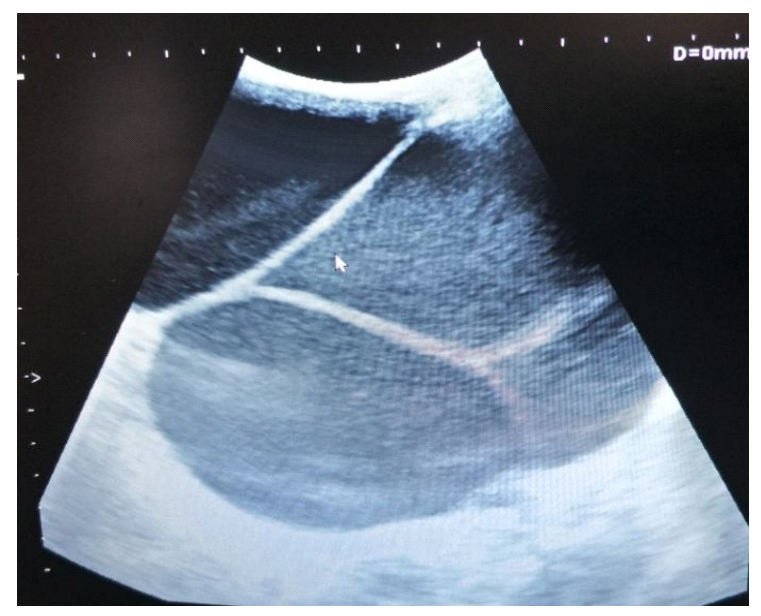

Fig 2: Cross section of anechoic to hypoechoic uterine sacculation in a spitz dog measuring $48 \mathrm{~mm}$ (average).

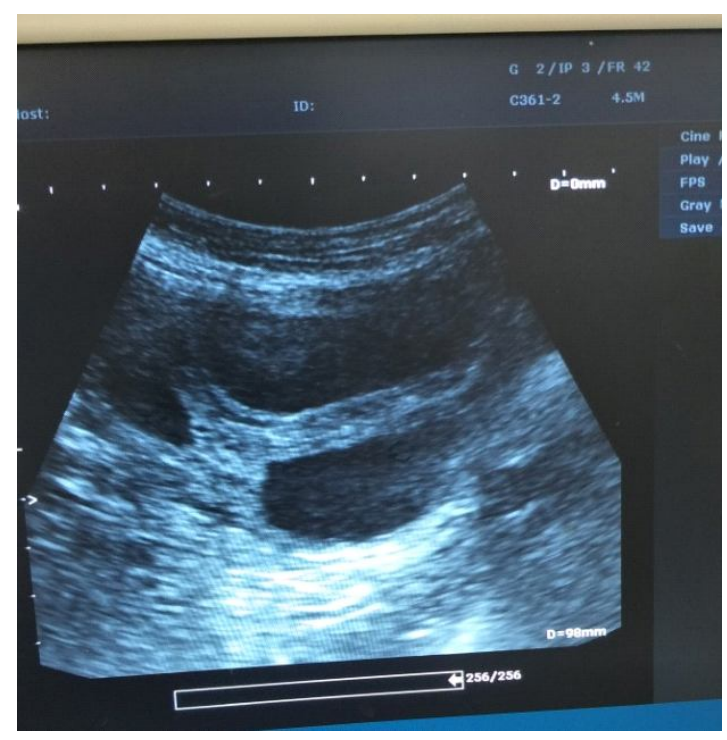

Fig 3: Longitudinal section of anechoic uterine sacculations measuring $10 \mathrm{~mm}$ (avaerage). 
cervix pyometra cases will take approximately 9 days. The combined effects of Methylergometrine and Cabergoline in severe cases of pyometra (uterine sacculation diameter of $>10 \mathrm{~mm}$ ) might take more than 10 days for complete recovery and evacuation of pus in severe toxemic cases when used in combination with supportive therapy (Fig 5 and 6). Kantharaj et al. (2018) reported the effects of methyl ergometrine drug alone on evacuation of pus in open

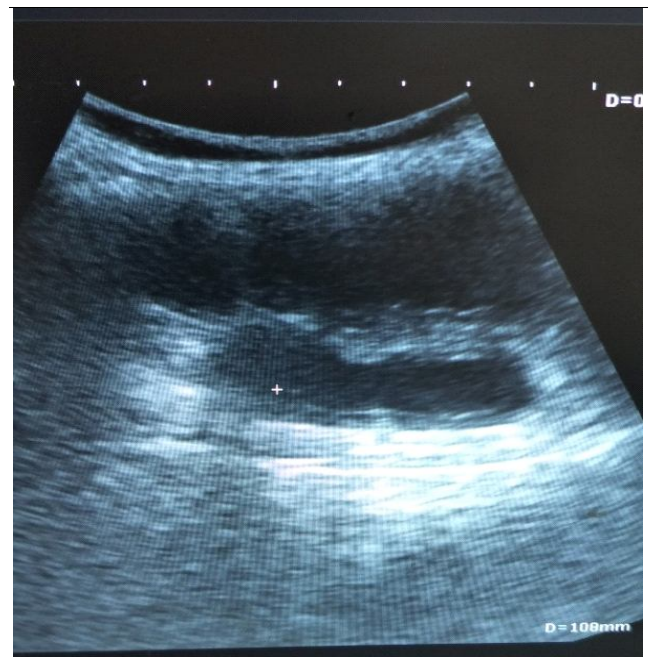

Fig 4: Longitudinal section of anechoic uterine sacculations measuring $5 \mathrm{~mm}$ (average).

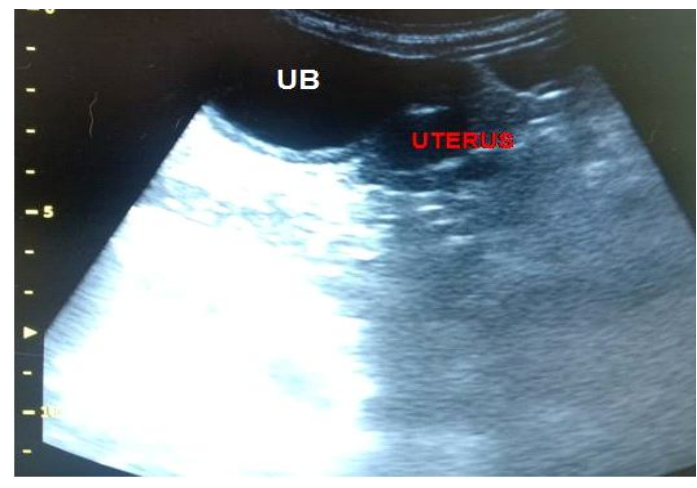

Fig 5: Cross section of Uterus showing lesser pus acccumulation in the uterus.

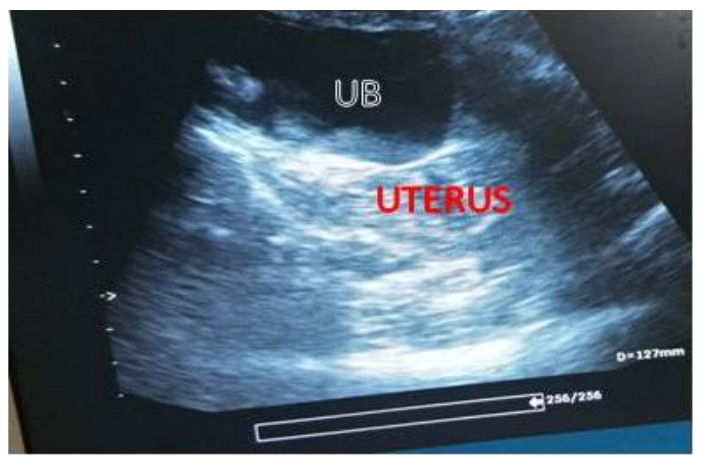

Fig 6: Complete evacuation of pus from uterus at day 14 of treatment in severe cases. pyometra cases; our study has a combination of Methylergometrine and Cabergoline therapy ensures evacuation of pus and luteolysis actions respectively (Verstegen et al., 2008). It can be concluded from our study evacuation of pus was achieved by ecbolic action of Methylergometrine and luteolytic mechanism induced by cabergoline. Further this treatment protocol was observed to have less side effects, less animal handling for administration of drugs, reduces the number of visits to the hospital when compared to the PG therapy.

\section{CONCLUSION}

In conclusion, either Methergine alone or in combination with cabergoline can be effective and safe for the treatment of open cervix pyometra in young and geriatric dogs unfit for traditional Ovariohysterectomy and in young dogs reproductive capability can be restored. The rationale behind this protocol was cabergoline ensures luteolysis and Methergine evacuates the pus safely even in over distended uterus without much complications as compared to Traditional Prostaglandins protocol. Nevertheless, careful case selection is important and medical treatment will ensure the complete recovery and prevents complications like Renal failure and death.

\section{ACKNOWLEDGEMENT}

We thank The Dean, Veterinary College and Research Institute (VCRI), Tirunelveli and The Director of Clinics, Tamil Nadu Veterinary and Animal Sciences University (TANUVAS) for providing necessary facilities for conduct of this case study and we also thank The Associate Professor and Head (i/c) Veterinary Clinical Complex (VCC), VCRI, Tirunelveli, for providing necessary inputs for conduct the case study.

\section{REFERENCES}

Andersen, V.R. and Farstad, W. (2006). Treatment of pyometra in the bitch: A survey among Norwegian small animal practitioners. Eur. J. companion anim. Pract. 16: 195-198.

Baithalu, R., Biswa, M., Chinmoy, M. and Lipismita, S. (2010). Canine Pyometra. Veterinary World. 3(7): 340-342

Burke, T.J. (1982). Prostaglandin F2 alpha in the treatment of pyometra-metritis. Vet Clin North Am Small Animal Practice. 107-109.

Corrada, Y., Arias, D., Rodriguez, R., Tortora, M. and Cobello. (2006). Combining dopamine agonist and prostaglandin agonist treatment of cystic endometrial hyperplasiapyometra complex in the bitch. Theriogenology. 1557-1559.

Dabhi, D.M. and Dhami, A.J. (2006). Serum urea, creatinine, cholesterol and protein profile in bitches with pyometra. Indian Veterinary Journal. 83: 1182-1185.

Kumar, P., Purohit, G.N., Barolia, Y.K., Yadav, S.P. and Bargujar, J. (2010). Clinical findings and medical therapy of pyometra in bitches. J. Canine Dev Res. 6: 79-82.

Kantharaj, K., Antoine, D., Murugavel, K., Shalini, G., Kunde, A., Hemalatha, H. and Raju, M.S. (2018). Treatment of open pyometra with methylergometrine in bitches. Indian Veterinary Journal. 95. 49-51. 
Rautela, R. and Katiyar, R. (2019). Review on canine pyometra, oxidative stress and current trends in diagnostics. Asian Pac. J. Reprod. 8(2): 45.

Sandhya, U.V. (2018). Biochemical Alterations in Canine Pyometra. Ind. J. Pure App. Biosci. 6: 199-201.

Shiju Simon, M., Ganesh, R., Arun Prasad, A. and Kumar, R.S. (2011). Incidence of pyometra in bitches-a survey of 278 cases. Tamilnadu J. Vet. Anim. Sci. 7: 252.
Shukla, S.P. (2012). Recent advances in canine pyometra. Indian J. Canine Pract. 4(1): 25-29.

Smith, F.O. (2006). Canine pyometra.Theriogenology. 66: 610-612. Verstegen, J., Dhaliwal, G., Verstegen-Onclin, K. (2008). Mucometra, cucstiv endometrial hyperplasia and pyometra in the bitch: Advances in treatment and assessment of future reproductive success. Theriogenology. 364-374. 